\title{
Proximal Sessile Polyps: Raised Expectations for the Detection of Flat Lesions
}

\author{
Pamela Lu ${ }^{1,2} \cdot$ Adam C. Fields $^{1} \cdot$ Nelya Melnitchouk ${ }^{1,2}$ \\ Published online: 4 July 2019 \\ ○) Springer Science+Business Media, LLC, part of Springer Nature 2019
}

\begin{abstract}
Although the use of colonoscopic screening programs has decreased the incidence of colorectal cancer and increased diagnoses of early stage disease [1], the benefit of screening in the prevention or early identification of disease in the proximal colon is inferior to that seen in the distal colon [2]. The efficacy of screening colonoscopy programs relies heavily on the skill of the endoscopist—both technically with respect to the ability to traverse and examine the entire colon, and the ability to accurately identify problematic lesions when present.
\end{abstract}

Serrated polyps are a particular subset of colonic lesions that have garnered increased attention in recent decades. The serrated pathway toward malignant transformation is associated with hypermethylation of $\mathrm{CpG}$ islands and $B R A F$ mutations. Serrated polyps are now thought to be responsible for $>15 \%$ of all colorectal cancers and significant contributors to the incidence of interval cancers [3, 4]. Despite the increasing awareness of these polyps and the importance of their detection for cancer prevention, the identification of such lesions can be difficult since proximal serrated polyps are often flat and have indistinct borders [3]. As such, the current literature reflects a wide range of proximal serrated polyp detection rates (PSPDR) between endoscopists, with a heterogenous body of literature with respect to the factors that may affect PSPDR detection variance.

In this issue of Digestive Diseases and Sciences, Mandaliya et al. [5] compare the rate of proximal serrated polyp detection in three different groups of physicians performing screening colonoscopies: academic gastroenterologists,

Nelya Melnitchouk

nmelnitchouk@bwh.harvard.edu

1 Division of General and Gastrointestinal Surgery, Brigham and Women's Hospital, Harvard Medical School, 75 Francis Street, Boston, MA 02115, USA

2 Department of Surgery, Center for Surgery and Public Health, Brigham and Women's Hospital, Harvard Medical School, Boston, MA, USA community gastroenterologists, and colorectal surgeons. In this single-institution retrospective review, all complete screening colonoscopies were performed on patients $>$ age 50 with good or excellent bowel preparation performed by 18 endoscopists between July 2015 and December 2017. A total of 2850 screening colonoscopies were included; the majority (53.4\%) were performed by six academic gastroenterologists, whereas $32.2 \%$ were performed by seven community gastroenterologists, and only $14.4 \%$ were performed by five colorectal surgeons. The authors found that academic gastroenterologists had significantly higher detection rates of both proximal serrated polyps and of sessile serrated adenomas, despite all three groups of endoscopists having no significant difference in the de facto standard for endoscopic quality, namely adenoma detection rates (ADR) (Table 1).

While this manuscript is the first to compare the detection rate of proximal serrated polyps between these three specific groups of physicians who perform screening colonoscopies, prior studies have evaluated the effect of different endoscopist characteristics on PSPDR with mixed results. For example, Sanaka et al. examined the differences in PSPDR among gastroenterologists, colorectal surgeons, and other endoscopists (primary care physicians/general surgeons). While they found no significant difference in PSPDR among groups of endoscopists, they did similarly report a wide variability in detection rates amid individual endoscopists [6]. In an evaluation of endoscopist characteristics and their effects on PSPDR, Sarvepalli et al. [4] found in multivariate analysis, adjusting for patient and procedural factors, that the only significant factors affecting PSPDR were the length of time since completing training and annual colonoscopy volume - physician specialty—also had no significant effect. In contrast, Parikh et al. [7] demonstrated that in the adjusted analysis, gastroenterologists had significantly higher PSPDR compared to non-gastroenterologists for screening colonoscopies. Importantly, prior studies compare cohorts of endoscopists that are more mixed-often combining general surgeons and colorectal surgeons, or all 
Table 1 Mean detection rates by endoscopist role (from [5])

\begin{tabular}{lcccc}
\hline & $\begin{array}{l}\text { Academic gastroenter- } \\
\text { ologists }(n=6)\end{array}$ & $\begin{array}{l}\text { Community gastroen- } \\
\text { terologists }(n=7)\end{array}$ & Colorectal surgeons $(n=5)$ & $P$ value \\
\hline Adenoma detection rate (ADR) & $40.3 \%(31.2-49.5)$ & $36.0 \%(30.0-44.4)$ & $39.6 \%(30.37-48.83)$ \\
Proximal adenoma detection rate (PADR) & $35.6 \%(12.7-58.6)$ & $24 \%(14.6-33.3)$ & $31.4 \%(18.9-43.8)$ \\
Sessile serrated adenoma detection rate (SSADR) & $3.8 \%(2.04-5.52)$ & $0.8 \%(0.03-1.63)$ & $0.8 \%(1.03-2.63)$ & 0.62 \\
Proximal serrated polyp detection rate (PSPDR) & $12.3 \%(7.04-17.42)$ & $5.4 \%(.03-8.74)$ & $4.5 \%(1.79-7.2)$ & 0.002 \\
\hline
\end{tabular}

non-gastroenterologists into a single group. Although the authors of the current study appropriately separate their endoscopists into three groups which may differ in training background, volume, and practice locations, their analysis is limited in that they did not adjust for other procedural or patient characteristics [5].

Many of these prior studies are limited by having data restricted to that of a single institution; this study is no different [5]. This is an important factor when considering the results of this study as it has been previously shown that there is wide variability on PSPDR at the endoscopy center level, suggesting that practice culture of an individual institution or education curriculum may impact the standard practices of its endoscopists [8]. Nonetheless, it also suggests that endoscopy center or program-based education may be effective. Although changes in attitudes toward recognizing the importance of proximal serrated polyps and colorectal cancer have led to the introduction of consensus guidelines regarding serrated polyps as recently as 2012, translation into practice may be lagging [9]. The authors of this paper suggest a minimum PSPDR of $9 \%$ based on the current literature as a benchmark metric, which many endoscopists fall below in the current literature [5]. While evidence-based benchmarks are important, equally important are suggestions on how to raise the standards of the national colonoscopy community to a new designated level. One step may be to widely develop endoscopy center or institutionally based educational programs; promisingly, Li et al. found that after one didactic education session focused on the identification and removal of sessile serrated polyps, gastroenterologists were able to significantly increase their PSPDR [10].

In conclusion, Mandaliya et al. [5] have shown that there is a significant difference in the PSPDR among academic gastroenterologists, community gastroenterologists, and colorectal surgeons. Their findings, in the context of the existing literature, suggest that the low and variable PSPDR among endoscopists is likely a result of a combination of differences in training, practice volume, and continuing education. It also highlights the need for future studies that identify effective tools or educational programs that can increase the average PSPDR among all endoscopists, especially those located in rural and community settings that provide services to patients with limited access to medical specialists.

\section{References}

1. Kahi CJ, Hewett DG, Norton DL, Eckert GJ, Rex DK. Prevalence and variable detection of proximal colon serrated polyps during screening colonoscopy. Clin Gastroenterol Hepatol. 2011;9:42-46.

2. Brenner H, Hoffmeister M, Arndt V, Stegmaier C, Altenhofen L, Haug U. Protection from right- and left-sided colorectal neoplasms after colonoscopy: population-based study. J Natl Cancer Inst. 2010;102:89-95.

3. Anderson JC. Pathogenesis and management of serrated polyps: current status and future directions. Gut Liver. 2014;8:582-589.

4. Sarvepalli S, Garber A, Rothberg MB, et al. Association of adenoma and proximal sessile serrated polyp detection rates with endoscopist characteristics. JAMA Surg. 2019. https://doi. org/10.1001/jamasurg.2019.0564.

5. Mandaliya R, Baig K, Barnhill M, et al. Significant variation in the detection rates of proximal serrated polyps among academic gastroenterologists, community gastroenterologists and colorectal surgeons in a single tertiary care center. Dig Dis Sci. (Epub ahead of print). https://doi.org/10.1007/s10620-019-05664-w.

6. Sanaka MR, Gohel T, Podugu A, et al. Adenoma and sessile serrated polyp detection rates: variation by patient sex and colonic segment but not specialty of the endoscopist. Dis Colon Rectum. 2014;57:1113-1119.

7. Parikh MP, Muthukuru S, Jobanputra Y, et al. Proximal sessile serrated adenomas are more prevalent in caucasians, and gastroenterologists are better than nongastroenterologists at their detection. Gastroenterol Res Pract. 2017;2017:6710931.

8. Payne SR, Church TR, Wandell M, et al. Endoscopic detection of proximal serrated lesions and pathologic identification of sessile serrated adenomas/polyps vary on the basis of center. Clin Gastroenterol Hepatol. 2014;12:1119-1126.

9. Crockett SD, Snover DC, Ahnen DJ, Baron JA. Sessile serrated adenomas: an evidence-based guide to management. Clin Gastroenterol Hepatol. 2015;13:e1.

10. Li D, Woolfrey J, Jiang SF, et al. Diagnosis and predictors of sessile serrated adenoma after educational training in a large, community-based, integrated healthcare setting. Gastrointest Endosc. 2018;87:e1.

Publisher's Note Springer Nature remains neutral with regard to jurisdictional claims in published maps and institutional affiliations. 